\title{
RECIPROCIDADES "OTRAS". El CONOCIMIENTO ANTROPOLÓGICO EN RETROSPECTIVA Y PROSPECTIVA EN LA AMAZONÍA ECUATORIANA
}

\author{
JAIRO TOCANCIPÁ-FALLA ${ }^{1}$
}

\begin{abstract}
RESUMEN
La producción antropológica supone una representación de un conocimiento, ajeno o familiarmente extrañado, que muchas veces oculta 0 no enseña el tipo de relaciones que establece el antropólogo con sus pares en campo. Nos acostumbramos, tal vez por la tendencia "objetivista", a hablar de las reciprocidades de los "otros" y no de "nuestras" reciprocidades implícitas o explicitas que sostenemos para ser aceptados en campo. Si bien en el Don de Marcel Mauss se examina la reciprocidad en "sociedades arcaicas", dicho planteamiento es revisado en este articulo para analizar las relaciones, que establece el/la antropólogo/a cuando recién llega al campo, pero con otras temporalidades, contextos y actores sociales. El caso de reflexión corresponde a un conjunto de experiencias vividas en la Amazonía ecuatoriana donde en diferentes contextos y escenarios, algunos líderes y comuneros de las nacionalidades sacaron a relucir reciprocidades no cumplidas por parte de antropólogos que llegaron a sus territorios en épocas pasadas. Se recomienda entonces una mayor consciencia y orientación con respecto a "otras reciprocidades" en el terreno, cuando se trata de avanzar en otros campos de investigación colaborativa.
\end{abstract}

\section{PALABRAS CLAVE}

Reciprocidad; Trabajo de Campo; Reflexividad; Antropología en el Ecuador

\section{"OUTRAS" RECIPROCIDADES. UMA RETROSPECTIVA DO CONHECIMENTO ANTROPOLÓGICO NA PERSPECTIVA DA AMAZÔNIA EQUATORIANA}

\begin{abstract}
RESUMO
A produção antropológica supõe uma representação do conhecimento, de familiaridade ou estranhamento, que muitas vezes oculta ou não ensina o tipo de relações que o antropólogo estabelece com seus pares em campo. Nós nos acostumamos, quem sabe pela influência "objetivista", a falar da reciprocidade do "outro" e não das "nossas" reciprocidades implícitas ou explícitas. Daquelas que precisamos sustentar para sermos aceitos no campo. Tomamos reciprocidade das "sociedades ditas primitivas" discutidas no "Ensaio sobre o Dom" de Marcel Mauss. Esta abordagem é revista nesse artigo para analisar as relações, que estabelece a / 0 antropólogo / a quando chega ao campo, mas com diferentes temporalidades, contextos e atores sociais. 0 caso que me conduz a essa reflexão vem de um conjunto de vivências na Amazônia equatoriana, onde, em diferentes contextos e cenários, alguns líderes e membros da comunidade de diferentes nacionalidades trouxeram reciprocidades insatisfeitas por antropólogos que vieram para os seus territórios em experiências passadas. Recomenda-se uma maior conscientização e orientação sobre "outras reciprocidades" no campo, para que possamos problematizar 0 avanço de outros campos de pesquisa colaborativa.
\end{abstract}

\author{
Palavras-Chave \\ Reciprocidade; Trabalho de campo; Reflexividade; Antropologia no Ecuador
}

\footnotetext{
1 Profesor Titular, Departamento de Antropología, Universidad del Cauca, Colombia/Becario
} Prometeo Senescyt, Universidad Estatal Amazónica (UEA), Ecuador. 


\title{
"OTHER'S" RECIPROCITIES. A RETROSPECTIVE OFANTHROPOLOGICAL KNOWLEDGE IN THE PERSPECTIVE OF EQUATORIAL AMAZONIA
}

\begin{abstract}
The anthropological production assumes a representation of knowledge, foreigner or familiarly misunderstood, that often hides or does not explain the kind of relations anthropologists establish with their interlocutors within the field. We have become accustomed, perhaps by the "objectivist" influence, to talk about the reciprocity of the "other" and not about "our" implicit or explicit reciprocity. Those we sustain to be accepted in the field. We have taken the concept of reciprocity, within the so-called primitive societies, discussed in Marcel Mauss's "Essay on the Gift". This approach is reviewed in this article, here we analyze the relationships anthropologists establish when he or she arrives in the field, considering different temporalities, contexts and social actors. The case that leads me to this reflection comes from a set of lived experiences in the Ecuadorian Amazon, where, in different contexts and settings, some leaders and members of the community from different nationalities brought dissatisfactions with reciprocities from anthropologists who came in the past to their territories. It is recommended greater awareness and guidance on "other reciprocities" in the field, so that we can problematize the advance of other fields of collaborative research.
\end{abstract}

\section{KEY WORDS}

Reciprocity; fieldwork; reflexivity; anthropology of Equator

\section{"AUTRES" RÉCIPROCITÉS. UNE RÉTROSPECTIVE DE LA CONNAISSANCE ANTHROPOLOGIQUE DANS LA PERSPECTIVE DE L'AMAZONIE ÉQUATORIAL}

\begin{abstract}
La production anthropologique suppose une représentation des connaissances, étrangères ou familiers, souvent cachés ou ne pas enseignées par le genre de relations que l'anthropologue établit avec ses interlocuteurs sur le terrain. Peut-être, nous nous sommes habitués par l'influence "objectiviste " à parler de la réciprocité de "l'autre» et non pas de "notre" propre réciprocité - soit elle implicite ou explicite. Il s'agit des réciprocités accordées pour qu'on soit accepté sur le terrain. Pour y comprendre, nous prenons le concept de réciprocité chez les « sociétés dites primitives » discutées dans le « Essai sur le don » de Marcel Mauss. Cette approche est utilisée ici pour analyser les relations établis par un /une anthropologue en arrivant sur le terrain, tout en considérant la temporalité, les contextes et les acteurs sociaux. Le cas empirique qui nous conduit à cette réflexion provient d'une série d'expériences dans l'Amazonie équatorienne, où, dans des contextes différents et des scénarios, des dirigeants et des membres de différentes communautés et nationalités ont mis en relief des réciprocités insatisfaits par les anthropologues qui sont entrés dans leur territoire. Nous recommandons une plus grande sensibilisation sur les " autres réciprocités " dans le terrain de recherche, afin que nous puissions discuter de l'avancement des autres domaines de recherche en collaboration.
\end{abstract}

\section{KEY WORDS}

Réciprocité; terrain de recherche; réflexivité; anthropologie en Équateur 


\section{INTRODUCCIÓN}

Generalmente la producción antropológica supone una representación de un conocimiento, ajeno o familiarmente extrañado, que muchas veces oculta o no hace evidente el tipo de relaciones que establece el antropólogo con sus pares en campo. Nos acostumbramos, tal vez por la tendencia objetivista a hablar de las reciprocidades de los "otros" y no de nuestras reciprocidades implícitas o explicitas que sostenemos para ser aceptados, y que calificamos como "reciprocidades otras". Si bien en el Don de Marcel Mauss (1990) se examina la reciprocidad en "sociedades arcaicas", dicho planteamiento es retomado en esta presentación para examinar las relaciones, que establece el antropólogo cuando recién llega a nuevos terrenos, sus temporalidades y acciones preventivas que establecen los actores sociales frente a la presencia de nuevos antropólogos e investigadores. El caso de reflexión corresponde a un conjunto de experiencias vividas en la amazonía ecuatoriana donde en diferentes contextos y escenarios, algunos líderes y comuneros de las nacionalidades sacaron a relucir reciprocidades no cumplidas por parte de antropólogos que llegaron a sus territorios en épocas pasadas. Este planteamiento es examinado y revisado a la luz de las implicaciones que tiene en la producción de conocimientos antropológicos y el mensaje que contiene en su reclamación para avanzar en una antropología más equitativa.

El texto se divide en siete secciones. En la primera se abordan dos tipos de representaciones, entre otras, sobre la amazonía y sus implicaciones para un análisis de las reciprocidades. En la segunda sección se abordan elementos del contexto examinado, en este caso la amazonía ecuatoriana, aspectos de territorio y dinámicas del poblamiento. La tercera sección revisa la idea de la amazonia como doble fuente de conocimiento, tanto para gobernantes, comerciantes y aventureros y particularmente en el caso de académicos e investigadores de distintos campos del conocimiento. Luego en una cuarta y quinta sección se aborda la noción de conocimiento antropológico en retrospectiva y su relación con el sistema de prestación total de la obra de Marcel Mauss (1990), el Don, y el conocimiento antropológico situado en prospectiva. Por último, se presentan algunas consideraciones finales sobre las implicaciones que tiene el desarrollo de reciprocidades incumplidas en contextos donde antropólogos en el pasado han actuado; al tiempo que se ofrecen algunos interrogantes para investigaciones posteriores.

\section{“TIERRA DE PROMISIÓN" Y “TIERRA DE PROMESAS INCUMPLIDAS": LAS REPRESENTACIONES DE LA AMAZONÍA ECUATORIANA}

Históricamente la Amazonía se reconoce como una vasta región de gran interés para misioneros, naturalistas, empresarios, y más recientemente aventureros, turistas y antropólogos, entre otros. Este variado interés todavía se mantiene hoy a través de diferentes 
imaginarios o representaciones ${ }^{2}$, algunas de las cuales datan desde hace más de una centuria mientras otras han surgido en décadas recientes ${ }^{3}$. En el caso particular de la cuenca amazónica ecuatoriana, deseamos contrastar por lo menos dos de ellas que denominaremos "La Amazonía como Tierra de promisión", y "La Amazonía como promesa incumplida". Cada una de estas representaciones guardan una relación estrecha y solo deseamos destacar su contenido, coexistencia y efecto en ciertos períodos de tiempo determinado.

Para empezar, la primera representación "La Amazonía como Tierra de promisión"también asociada con expresiones tales como "Tierras del Dorado", "Tierra prometida"- es convergente con aquellas impresiones que misioneros, administradores y colonos ofrecieron desde hace centurias al hacer su ingreso en la región, identificando la selva amazónica como fuente de tesoros y recursos escondidos todavía por ser explorados y aprovechados. Así, los metales, como el oro, constituyeron una de las motivaciones principales para incursionar en la amazonía, entre otros recursos como fibras (pita), canela, cacao silvestre, y resinas que podían extraerse (TAYLOR, 1999, p. 188-256); motivaciones que, si bien se plantearon desde el siglo XVI, todavía prevalecen con diferentes intensidades en la actualidad ${ }^{4}$. La apertura de vías y sus justificaciones también evocan este tipo de representación de "Tierra de promisión", a través de las cuales y como lo señalaba un exgobernador de la gobernación del Oriente a comienzos del siglo XX, se busca el "adelanto y progreso de esta inmensa provincia tesoro de la riqueza nacional" (HURTADO, 1899b, p.2). Un colono que también conocía la región amazónica, especialmente la conocida hoy provincia de Pastaza, aleccionaba a sus amigos para que se colonizara la amazonía pues allí "existen riquezas abundantes, una exhuberante selva vegetal, son tierras para la agricultura, se puede vivir de la caza y de la pesca, el oro se encuentra en la arena de los ríos." (ZAMORA, 2009a). Para esta época también un exgobernador de la Gobernación del Oriente, Enrique Z Hurtado, haciendo eco de lo dicho por muchos viajeros de la época, señalaba que "El feliz porvenir y la futura grandeza del Ecuador se hallan vinculados

\footnotetext{
${ }^{2} \mathrm{Al}$ referirnos a representaciones aludimos a representaciones sociales, las cuales se entienden como esquemas mentales proyectados que dan sentidos significativos y que pueden servir de orientaciones no solo para el pensamiento, sino también para la acción. Tales esquemas mentales son validados por una colectividad en la cual se comparten valores, sentimientos, lenguaje y cultura, como ocurre con la idea de identidad nacional. Ellas pueden ser visuales, auditivas, corporales, etc. (ANDERSON, 1996; DUVEEN; LLOYD, 1990; JENKINS, 1996; POOLE, 1999). Existen otras representaciones sociales de las cuales no nos ocuparemos en este trabajo, como aquella referida a la amazonía como espacio de terror y sanación (Cfr., por ejemplo, TAUSSIG 2002).
}

${ }^{3}$ El Escudo de armas de la provincia de Pastaza, y esculturas-fuentes como la mukawa-vasija de barro típica en la tradición kichwa, inaugurada el 12 de mayo de 1987-y la del encuentro colonial -donde aparece un colono y un nativo compartiendo un paraguas para resguardarse de la lluvia, s.f.-, ubicadas en el centro de Puyo sobre la calle Ceslao Marín, son un ejemplo de tales representaciones como proyecciones y memoria de las relaciones interculturales, pero también de los valores propios de la amazonía (ZAMORA, 2009b).

\footnotetext{
${ }^{4}$ Hace poco un amigo waorani, me comentó de la posibilidad de entrar en asocio con un par de señores que deseaban explorar y explotar oro en su territorio.
} 
en la provincia del Oriente" (HURTADO, 1899a, p.4). Podría afirmarse que esta primera representación de la amazonía como territorios ignotos llenos de riqueza por ser exploradas y explotadas sigue siendo una constante histórica que desde el pasado ha trascendido en distintos medios, especialmente en el ámbito de la formación de los estados nacionales y como veremos más adelante, en relación con el conocimiento amazónico.

Ahora bien, y siguiendo con nuestra trayectoria, ya bien entrado el siglo XX, algunas riquezas como el oro fueron sustituidos por los hidrocarburos. En 1920 la Compañía Leonard obtuvo los derechos de exploración y explotación y una concesión de dos millones y medio de hectáreas para trabajar en la amazonía, y entre cuyos beneficios se planteó la apertura de la vía Baños-Puyo (ZAMORA, 2004, p. 31), la cual fue culminada en 1947, y que terminaría conectando a una provincia andina (Tumburahua) con otra amazónica (Pastaza). Para 1937, el gobierno ecuatoriano canceló el contrato con esta compañía y estableció uno nuevo con la Royal Dutch Shell Oil Company a la cual se le concedieron unas diez millones de hectáreas (Ibid.,32) y que a su llegada se convertiría en un importante centro de comunicación aérea desde el piedemonte andino hacia la amazonía y posteriormente en un centro poblado ubicado a solo 10 minutos de Puyo, capital de la provincia de Pastaza.

Es en este contexto que una segunda representación de la "Amazonía como promesa incumplida" se hace visible. Ciertamente, en 1948 ante el hecho de que la Shell despide en pocos días a 900 trabajadores, el entonces presidente del Ecuador Galo Plaza Lasso, declara que "El Oriente es un mito", para indicar que se trata de un vasto territorio de ilusiones no cumplidas. En términos contemporáneos, Zamora lo expresa así: "La explotación petrolera ha creado una especie de 'falsa economía' en la zona, toda vez que con el inicio de la explotación petrolera, se generó en su entorno muchas expectativas de 'empleo y desarrollo', situación que no es real."(ZAMORA, 2004, p. 33).

Pero este desaliento, y que se evoca en este tipo de representaciones, puede ser pasajero o transitorio, al menos desde una perspectiva de manejo político. Un poco más de una década después, el entonces presidente Velasco Ibarra declaró que el "Ecuador ha sido, es y será el país amazónico" (WHITTEN, 1976, p. 239; mi traducción), para indicar que como región sería objeto de inversiones extranjeras, asociadas también con la explotación de petróleo entre otras industrias. Algunas de estas compañías buscaban ampliar la frontera agrícola con cultivos de caña -inicialmente empresarios brasileños en 1935-y posteriormente también se evidenció décadas después con el caso del Té -especialmente empresarios británicos y estadounidenses en 1964- y la ganadería 5 .

\footnotetext{
${ }^{5}$ El complejo de la ganadería, como lo denominó Whitten, constituyó una de las actividades pecuarias que los colonos e indígenas siguieron en atención al llamado que el Presidente Guillermo Rodríguez Lara hiciera en 1972 sobre la importancia de mejorar la infraestructura y acelerar la producción a pequeña escala y hacer más eficiente el uso de las tierras. A la par, este complejo ganadero se vio
} 
Podría afirmarse que estos dos tipos de representaciones, "La Amazonía como Tierra de promisión" y "La Amazonía como promesa incumplida" son coexistentes y alternativas en su manifestación. Pero igualmente a lo largo de los años y más en la actualidad, mantienen una tensión que igualmente expresan el poder de lo intocado y de la necesidad imperiosa de su transformación para el beneficio del pueblo ecuatoriano, como acontece con el gobierno vigente de Rafael Correa y los preceptos establecidos en su Plan de gobierno del "Buen Vivir". Es en el seno de esta tensión que deseamos enmarcar la retrospectiva y prospectiva del conocimiento antropológico que se ha logrado avizorar a partir de una breve experiencia de campo de más de un año en la amazonía ecuatoriana. Antes de entrar en materia es conveniente sumar otros aspectos del contexto amazónico.

\section{LA AMAZONÍA ECUATORIANA: ALGUNOS ELEMENTOS DEL CONTEXTO}

Actualmente al referirnos a la amazonía acuden a nuestra mente una buena cantidad de adjetivos y superlativos que aluden a su magnificencia y biodiversidad. Calificada con simbolismos como "el pulmón del mundo", "el mundo de la biodiversidad" o "la gran reserva de la biosfera" o "la mayor reserva biodiversa del mundo", etc. la amazonía se asocia con nueve países de la denominada cuenca amazónica: Surinam, Guyana, Guyana Francesa, Venezuela, Brasil, Colombia, Ecuador, Perú, y Bolivia. Su extensión se estima en 7.8 millones de km2 donde habitan 44 millones de personas, de las cuales 2.6 millones corresponden a poblaciones nativas con más de 390 pueblos étnicos o nacionalidades (NEVES; PETERSEN; BARTONE; SILVA, 2003) ${ }^{6}$. Brasil y Perú ostentan la mayor extensión de la Amazonía. Si bien existen aspectos comunes entre los países que hacen parte de la gran cuenca amazónica, también es cierto que existen diferencias ecosistémicas como las interrelaciones que se mantienen con los Antes (caso de Colombia, Ecuador, Perú y Bolivia) o con el océano Atlántico (caso de Venezuela, Surinam, Guyanas y Brasil). Antes de introducirnos en el caso de la cuenca amazónica ecuatoriana, es oportuno ofrecer algunos datos básicos del contexto más general.

Ecuador es un país de amplios y variados contrastes. El censo de 2010 arrojó una población de 14.451 .115 habitantes distribuidos en 24 provincias o departamentos con

\footnotetext{
también acentuado en parte por la relación que tuvo esta actividad pecuaria con el cultivo del maíz que, asociado con pastos, eran rentados a colonizadores en el período postcosecha; y por los ingresos que muchos comuneros obtuvieron al trabajar en la explotación petrolera los cuales fueron también orientados hacia la compra de ganado (Ver WHITTEN, 1976, p. 247, 252, 266, 264)

${ }^{6}$ En 1998, la Confederación Nacional de Indígenas del Ecuador (CONAIE) empezó a referirse al concepto de Nacionalidades en el contexto de la ratificación del Convenio 169 de la Organización Internacional del Trabajo (OIT), el cual fue aprobado ese mismo año en el Congreso a través de la Comisión de Asuntos Indígenas. Este reconocimiento fue parte de la misma lucha del movimiento indígena unido que terminó consagrado a nivel constitucional en el año 2008 y que a partir del cual se logró el reconocimiento por parte del Estado ecuatoriano a las nacionalidades amazónicas de más de un millón de hectáreas (BECKER, 2015; SAWYER, 2004).
} 
diferentes ecosistemas: 5 sobre la costa del pacífico y un archipiélago, 12 en los valles interandinos y andes centrales y 6 en la cuenca amazónica. Sobre un vasto territorio de 255.587 Kms2, el 45\% (116.604 Kms2) corresponden a la cuenca amazónica, el 27\% (71.013 Kms2) a los Andes y valles interandinos y el 26\% (67.969 Kms2) a la Costa Pacífica, aproximadamente. Esta distribución territorial, sin embargo, no se correlaciona con la densidad poblacional existente en el país. Mientras el 95\% de la población se ubica en un poco más del 50\% del territorio -Costa Pacífica, Andes y valles interandinos- el 5\% de la población restante se localiza en el $45 \%$ de lo que queda del territorio, en particular la cuenca amazónica. La densidad de la población por Km2 y por ecosistema, igualmente lo confirma: Andes y valles interandinos (96 habitantes por Km2), Costa (89 habitantes por Km2) y Amazonía (7 habitantes por Km2) (INES, 2015).

En el caso de la Región Amazónica Ecuatoriana (RAE), se estima que existe una población indígena de unos 225.000 habitantes (INES, 2015), dispersa a lo largo de las provincias mencionadas. En la actualidad (2016), de las 15 nacionalidades existentes en el Ecuador, 11 de ellas están localizadas en la cuenca amazónica y en límites con Perú y Colombia, a saber: Al' cofán, achuar, andwa, kichwa, sapara, siona, siona-secoya, shiwiar, shuar, waodani y quijos. En particular, estas nacionalidades se encuentran ubicadas a lo largo de las 6 provincias amazónicas del Ecuador: Sucumbíos, Orellana, Napo, Pastaza, Morona Santiago y Zamora Chinchipe (Ver Mapa No 1). Para el caso que examinaremos aquí nos enfocaremos en Pastaza, la provincia más extensa del Ecuador con 83.933 habitantes distribuidos en 29.641 Kms2, lo que equivale a una densidad poblacional de 3 habitantes por Km2. Igualmente se trata de la provincia más grande del Ecuador, donde habitan 7 de las 15 nacionalidades existentes en el país: achuar, andwa, kichwa, sapara, shiwiar, shuar, waodani.

Actualmente estas nacionalidades se encuentran agrupadas en organizaciones como la Confederación de Nacionalidades Indígenas de la Amazonía Ecuatoriana (CONFENIAE). Con respecto a estas nacionalidades, la documentación etnográfica e histórica es variable y disímil entre sí, pues mientras existe una literatura apreciable en el caso de los kichwa-grupo más numeroso de la amazonía ecuatoriana- y los achuar, shuar, y waorani, en el caso de los andwa, shiwiar y sapara, las referencias son escasas.

Igualmente, es en décadas recientes que las investigaciones antropológicas se han dinamizado un poco más. En lo que sigue y para referirnos a un conocimiento antropológico en retrospectiva y prospectiva, examinaremos algunas implicaciones que ha tenido el concepto de reciprocidad para las investigaciones presentes y futuras en la amazonía ecuatoriana, aunque potencialmente también se pueden hacer extensivas a otras latitudes. 


\section{LA AMAZONÍA COMO DOBLE FUENTE PRIMARIA DE CONOCIMIENTO}

Paralelo a las dos formas de representación ya señaladas con respecto a los valores que evoca la amazonía, en décadas recientes se han sumado otras formas de representación que valoran este vasto territorio como fuente de conocimiento. Expresiones relativamente recientes como bioconocimiento, conocimiento y saberes ancestrales aparecen en publicaciones recientes y especialmente en documentos de política social que establece el Estado ecuatoriano. En la Constitución del Ecuador (2008), por ejemplo, la expresión "conocimiento" aparece vinculada con los derechos de las comunidades, pueblos y nacionalidades en lo referido a "Mantener, proteger y desarrollar los conocimientos colectivos" y su prohibición en sus formas de apropiación (CONSTITUYENTE, 2008, p.42, ver también Artículos 322 y 402); además de reconocer y promocionar el uso del conocimiento medicinal (Art. 363, numeral 4 y Art. 388), y el rescate del conocimiento ancestral (Art. 388) ${ }^{7}$. En el ámbito de diferentes campos de conocimiento como la biología, ciencias ambientales, y antropología, la amazonía ha sido un poderoso referente que invita a escudriñar y reflexionar sobre la relación entre el conocimiento y los procesos adaptativos de los pueblos amazónicos. Son escasas las referencias donde no se aluda a ese potencial acervo de interrelaciones. En el caso de la antropología, por mencionar algunas referencias, el trabajo clásico de Levi-Strauss en el Brasil con los nambikwara, Reichel-Dolmattoff con los desana en Colombia, Descola con los achuar, Uzendoski y los Whitten con los kichwa de Napo y Pastaza en el Ecuador respectivamente, reflejan en buena medida la importancia de estas interrelaciones entre conocimiento de los pueblos y procesos adaptativos, entre otros temas.

Algunas de estas referencias sobre el conocimiento de los pueblos amazónicos, como en el caso del trabajo de Levi-Strauss (1979), sin embargo, están inscritas más bien en una perspectiva objetivista ${ }^{8}$, en cuya perspectiva se representa buena parte de la realidad social. Esta representación objetiva de la realidad social, sin embargo, ha sido cuestionada en décadas recientes desde la crítica literaria y la linguística por su carácter totalitario, omnisciente y ficcional (CLIFFORD, 1986; THORNTON, 1988); crítica que busca posicionar otras perspectivas en la comprensión de la realidad social, donde el papel y prefiguraciones del etnógrafo en la

\footnotetext{
${ }^{7}$ Las diferencias en el uso de conocimiento y saberes ancestrales no es clara en la Constitución.

${ }^{8}$ Para una discusión sobre modos de conocimiento en los 1970, ver BOURDIEU $(1977 ; 1982)$.
} 
estructuración de dicha representación y la dimensión política como componentes centrales en la discusión, aparecen de manera destacada.

Mapa 1. Nacionalidades de la cuenca amazónica ecuatoriana

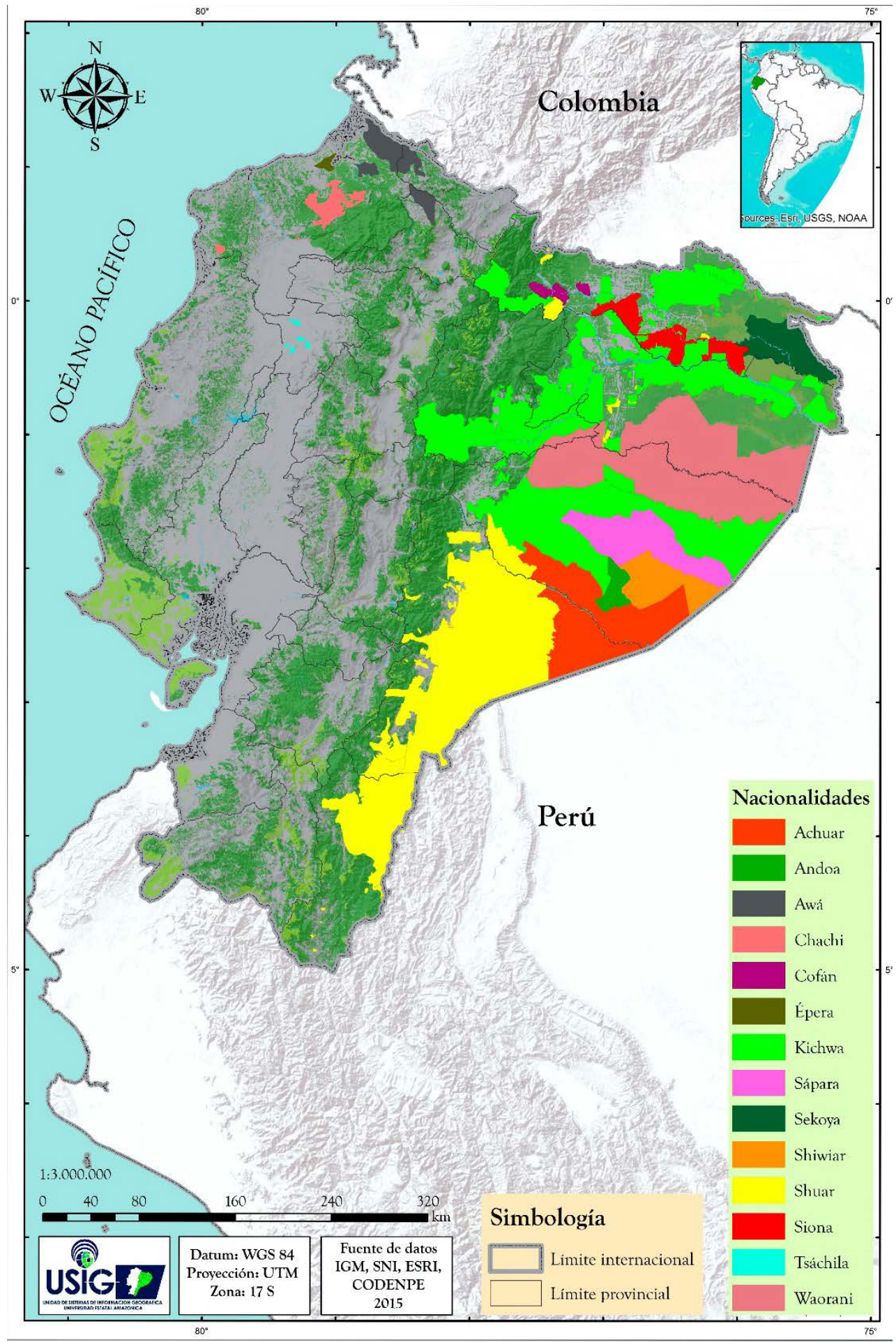

Fonte:Unidad deSistemas de Information Geografica - Universidad Estatal Amazonica 
Con todo, la crítica que se hace a la etnografía tiene unos alcances a nivel teórico pero también limitaciones en su receptividad; muchos antropólogos profesionales e investigadores en la actualidad continúan ensayando las ideas básicas de los manuales etnográficos y la práctica etnográfica todavía persiste. Esta continuidad se puede apreciar en lugares donde el imaginario antropológico se vuelve destacado y recurrente como es el caso de la amazonía donde lo exótico y lo intocado aparecen de manera atrayente para muchos aventureros, misioneros o empresarios. Desde este ángulo, la amazonía puede ser apreciada en doble vía: como fuente de conocimiento antropológico destacado que contrasta con aquél interés que han motivado a otros estudiosos como biólogos, botánicos y naturalistas, entre otros, y donde buscan saciar la sed de ese conocimiento ancestral y esa riqueza biodiversa existente allí; pero también aparece la representación de una amazonía como receptora de conocimiento donde las ideas transformadoras agenciadas por el Estado, las empresas, multinacionales, universidades -y por supuesto, también investigadores y académicos- y Ong entre otros actores, buscan germinar para dar lugar a otro mundo imaginado que supere la condición agreste y salvaje que deriva de su impresión paisajística. Es claro que ambas condiciones son coexistentes y correspondientes tal como se puede apreciar en la actualidad con la política del Estado ecuatoriano que aboga por hacer valer el conocimiento ancestral dentro de un Estado reconocidamente plurinacional e intercultural, pero al tiempo que se aplica una política de transformación de la matriz productiva que se consagraba en el Plan Nacional del Buen Vivir (2009-2013) ${ }^{9}$. Si bien teóricamente estas dos ideas pueden equilibrarse, para autores como Weiss y Bustamante (2008, p.19), en la práctica, y con respecto a este último Plan "una visión del crecimiento económico" cara a cara con "la sustentabilidad ambiental" todavía se mantienen como dos "conceptos antagónicos". Pero las paradojas en términos del conocimiento no son necesariamente peculiares a las políticas del Estado. En el ámbito del conocimiento en las ciencias sociales y las humanidades, y en especial la antropología, es una condición fundamental para comprender las complejidades de la trama en las cuales se ve envuelto el antropólogo en su quehacer disciplinario con otros actores sociales.

\section{EL CONOCIMIENTO ANTROPOLÓGICO EN RETROSPECTIVA Y EL SISTEMA DE PRESTACIÓN TOTAL DE MAUSS}

Mi experiencia personal en la amazonía es reciente. Si bien había tenido un acercamiento esporádico con algunas comunidades amazónicas en Colombia, es solo en el

\footnotetext{
${ }^{9}$ A partir del año 2007, se inició el primer Plan Nacional de Desarrollo (2007-2010) del gobierno ecuatoriano actual que fue presentado como propuesta en las elecciones de 2006. Posteriormente vino un segundo Plan Nacional para el Desarrollo conocido como "El Buen Vivir" a partir de la Constitución de Montecristi (2008) y que se presentó para el período 2009-2013. Finalmente, en la actualidad se encuentra El Plan Nacional para el "Buen Vivir" (2013-2017), que corresponde al tercer período del mandatario Rafael Correa (SENPLADES, 2013).
} 
2014 cuando, a través del programa Prometeo de la Secretaría Nacional para la Educación Superior, la Ciencia, la Tecnología y la Innovación (Senescyt) junto a la Universidad Estatal Amazónica (UEA) me conceden una beca de investigación para indagar sobre el conocimiento y los cambios tecnológicos en la producción alfarera de una comunidad kichwa de la provincia de Pastaza. Esta experiencia que se extendió por un poco más de un año, abarcando tres períodos de estancia -enero-febrero 2014, julio-diciembre 2015 y enero-julio 2015-, también involucró un trabajo sostenido de relacionamiento entre la UEA y las 7 nacionalidades que tienen presencia en Pastaza. Se trataba de inaugurar una relación sostenida, en un diálogo abierto y franco, que permitiera generar un clima de trabajo mancomunado alrededor de temáticas afines o comunes a las nacionalidades y a la misma universidad. A junio de 2015, el trabajo iniciado en el mes de enero de 2014 se fue consolidando a través de seis mesas de trabajo entre estos dos actores, la organización de un foro internacional sobre "Saberes ancestrales e interculturalidad" y el acompañamiento por parte de la UEA a jóvenes de las nacionalidades en el proceso de ingreso a la Universidad. Cada uno de estos escenarios posibilitó establecer los cimientos de una relación inédita entre una institución de Educación Superior y un conjunto de siete nacionalidades de pueblos indígenas presentes en la cuenca amazónica.

Es en este contexto, que deseo introducir aquí el problema que nos concierne sobre el conocimiento antropológico en retrospectiva, y que alude básicamente al menos a la producción antropológica representada en libros, textos y artículos mediante la cual se divulgan aspectos de la vida social y cultural de las sociedades amazónicas en diferentes períodos de tiempo y que representan tanto para las comunidades académicas como para los mismos pueblos indígenas referentes de aprendizaje-enseñanza, poder y prestigio.

La emergencia de esta situación me fue revelada a comienzos de las visitas realizadas entre enero y febrero de 2014 y posteriormente en julio y agosto de ese mismo año, cuando al presentarme ante los líderes de las diferentes nacionalidades acudían expresiones reiterativas tales como "aquí vienen los antropólogos, entrevistan a nuestros ancianos y luego escriben libros con los que se hacen famosos y hacen dinero". O "aquí viene gente, investigan, y luego se llevan el conocimiento y no dejan nada en nuestras comunidades" ${ }^{10}$. Al comienzo, cuando registré estas impresiones, las tomé en primera instancia como una primera experiencia que algunos colegas habían tenido en sus comienzos de sus carreras, especialmente en la década de los 1970, y que habían dejado un legado de manera inadvertida. Sin embargo, luego al adentrarme más en las expresiones y tratarles de dar un sentido mayor,

\footnotetext{
${ }^{10}$ En otros lugares estas representaciones también son recurrentes como aconteció con una colega quien al inicio de su investigación antropológica como estudiante de pregrado con los dule en Panamá, recibió de entrada la expresión: "Ustedes los antropólogos siempre se quedan con la carne y nosotros con el hueso".
} 
noté que habían otras posibilidades no excluyentes. Una de estas posibilidades era entender tales expresiones como un dictum-una declaración dada con cierta autoridad sobre un asunto particular pero que se convertía casi en una fórmula- que buscaba establecer los términos de una negociación o acuerdo sobre la razón o motivo de la visita establecida por el recién llegado. En principio se trataba de una forma de reciprocidad, pues el líder que la anunciaba casi que pedía explicaciones a cambio, frente a ese dictum que no podía ser equivalente en su enunciación; es decir, no se podía decir casi lo mismo que se quería hacer una investigación pero que los resultados no serían compartidos con las nacionalidades, etc.

Otra posibilidad interpretativa, y que contextualizaba la producción antropológica, ега que las referencias aludían a un pasado relativamente distante cuando algunos antropólogos, seguramente los primeros en llegar a ciertas áreas de la amazonía, iniciaron un proceso de trabajo y producción antropológica por más de tres décadas (e.g. los Whitten y Descola entre otros) y justo cuando ideas como derechos de propiedad intelectual o derechos colectivos no estaban en boga por aquella época. Derivado de esta última interpretación estaba el hecho de que el conocimiento elaborado por colegas que antecedían la estancia en esta parte de la amazonía no se apartaba de un continuum histórico de representaciones sucesivas sobre lo que hacen los antropólogos, lo que producen y el posible impacto que tiene lo que hacen. Estas implicaciones estaban mediadas igualmente por un problema generacional pues mientras algunos líderes mayores estaban familiarizados con los antropólogos iniciales, las nuevas generaciones los veían como extraños tanto en sus personalidades como en los productos resultados de su estancia en décadas anteriores. Se podría afirmar que la experiencia pasada de los antropólogos les servía a las nuevas generaciones para contextualizar el problema del conocimiento en el presente. Una de las lecciones era justamente compartir la experiencia con el antropólogo, aprender con él y compartir los resultados obtenidos de dicha experiencia y favorecerse del prestigio y poder que dimana de los libros, videos, fotografías, etc. En este contexto conviene introducir una pregunta: ¿desde el punto de vista teórico qué tipo de lecturas podemos obtener de la idea del conocimiento y experiencia que nuestros antecesores prohijaron frente a los interlocutores nativos de la época?

Una aproximación a una respuesta implica hacer una relectura del trabajo de Marcel Mauss, quien propuso la idea de "sistemas de prestaciones totales"11 la cual invoca el concepto de reciprocidad presente en todas las sociedades. Básicamente, y en términos de este autor, "se trata de un sistema en el cual los individuos y los grupos intercambian todo entre sí,

\footnotetext{
${ }^{11}$ En la traducción de la obra de Mauss, y en particular la introducción Levi-Strauss se alude a actos sociales totales, y que luego tiene que justificarse como parte de un sistema, categoría que incorpora el carácter integral de la idea. Por ello seguiremos aquí la traducción de "sistema de prestaciones totales".
} 
constituyendo así el sistema más antiguo de la economía y la ley que podamos encontrar o del cual se pueda concebir. Forma la base a través de la cual la moralidad del intercambio -a través del don-fluye." (MAUSS, 1990, p. 70; mi traducción). En la introducción de la obra de Mauss, Levi Strauss señala tres aspectos destacados en esta noción: lo social, la experiencia individual y el sistema de interpretación que da cuenta de los aspectos físicos, psíquicos y sociólogicos; tarea que se encarga la antropología. Estos aspectos a su vez sugieren una dualidad de categorías contrapuestas pero complementarias y contrastantes: Lo social-individual y lo físico-psíquico.

Ahora bien, la manera como Mauss (1990) introduce esta noción asocia la idea de cierta totalidad que se basa en dos obligaciones, la de dar у recibir. Si bien sus descripciones se fundamentaron en términos comparativos entre diferentes sociedades, cabe llamar la atención sobre un aspecto que entraña dicha noción y que vincula las discusiones que actualmente ocupan a muchos antropólogos, a saber: el papel y compromiso, implícito o explícito, que asume el investigador frente al grupo que lo acoge. Justamente la crítica a la etnografía convencional que plantearon varios investigadores en América Latina y en Estados Unidos en la década de los 1970 y 1980 respectivamente, se fundamentó en esta aparente "invisibilidad" del antropólogo que actuaba en función de una causa académica, al tiempo que ignoraba su propia condición y la de los actores sociales con quienes estaba interactuando.

Sin embargo, deseo destacar que esta imputación concedida a este tipo de etnografía clásica convencional, no se percató de algunas ideas antecesoras planteadas por Mauss y refrendadas por Lévi-Strauss (1979) y que refieren a la importancia de la condición y situación del sujeto cognoscente en la escena cultural; consideración que ha contribuido en la fundamentación de ideas como "reflexividad" o "auto-antropología" (RAPPORT; OVERING 2007; WOOLGAR 1996). En particular, en varios pasajes de la introducción a la obra de Mauss, Levi-Strauss lo deja apenas planteado en la discusión. Uno de estos fragmentos señala, por ejemplo, que en la noción de sistema de prestación total, su comprensión no es solo un referente objetivo de la realidad social per se sino que en ella está presente el mismo etnógrafo en su propia condición social: "Que el acto social sea total no sólo significa que todo aquello que se observa forma parte de la observación, sino también, y sobre todo, que en una ciencia en que el observador es de la misma naturaleza que su objeto, éste es también parte integrante de su observación" (LÉVI-STRAUSS 1971; 1979, p. 25). Como tal, entonces la interacción que establece el antropólogo, como profesional-investigador y como sujeto, con sus pares "otros" -léanse los colaboradores o inicialmente como fueron llamados en términos objetivistas los "informantes"- plantea un problema de carácter comunicacional entre individuos que invoca múltiples dimensiones como lo social-individual y viceversa; y lo consciente-inconsciente y viceversa. Desde esta última perspectiva, y en especial en la "comprensión de las formas inconscientes de la actividad del espíritu”, Levi Strauss sugiere que 
aparece un problema que articula la subjetivación y la investigación etnológica: "el de encontrar una comunicación tanto entre el yo subjetivo y el yo objetivo, como entre el yo objetivo y el otro subjetivo" (LÉVI-STRAUSS 1971; 1979, p. 28). Es este marco referencial introductorio en el análisis de la obra de Mauss, conviene introducir la pregunta de cómo examinar los reparos que muchos líderes de la amazonía ecuatoriana me hicieron a mi llegada en calidad de antropólogo. Varias cuestiones al respecto merecen plantearse.

En primer lugar, es claro que en una fase inicial para la década de los 1960 y 1970 cuando llegan algunos antropólogos a la amazonia ecuatoriana la idea de reflexividad y auto antropología apenas estaba emergiendo en el horizonte de las discusiones. En la lectura de algunas de estas obras como la de los Whitten, Descola e incluso recientemente en la de Uzendoski -este último casado con una kichwa-, las referencias a una autoconsciencia sobre las implicaciones de sus trabajos de investigación en el presente etnográfico no son muy bien definidas. Más bien prevalece una relación directa con la necesidad de producir un conocimiento antropológico propio de nuestra disciplina. A la distancia del tiempo, esta apreciación no implica una subestimación de la importancia del trabajo etnográfico realizado por estos colegas, y más en los momentos actuales, sino que busca elucidar una situación histórica que a todas luces ni para el antropólogo, ni para las nacionalidades y pueblos indígenas en su momento constituía una pregunta o un problema de indagación. Es sólo décadas después, cuando las interacciones de las nacionalidades con diferentes actores y cuando las publicaciones de los antropólogos empiezan a sobresalir en distintos ámbitos en particular con respecto a la producción intelectual y su potencial prestigio y con la emergencia de los derechos de propiedad intelectual, que la obra de los antropólogos empieza a ser revisada en su momento actual y futuro. Desde esta perspectiva, las relaciones que los antropólogos establecieron en el pasado con su pares "otros" siguen siendo dinámicas y sostenidas a través del tiempo, a la vez que sirven de referente en las negociaciones de ingreso al terreno por parte de nuevos practicantes en la disciplina.

En segundo lugar, y con particular referencia a la idea de reciprocidad del trabajo que realizan los antropólogos, existe la idea de que el poder y reconocimiento que el antropólogo tiene al publicar un libro o un trabajo determinado pueda ser compartido con sus interlocutores. Así, las alusiones reiteradas a las promesas incumplidas de los antropólogos por dar a conocer, retribuir y retornar sus trabajos antropológicos a sus contrapartes en el terreno puede tener una interpretación de establecer los términos de una relación más recíproca y equitativa con lo que se tiene previsto realizar en campo. En esta interpretación, se tienen al menos dos variantes. Una es la validación por parte de los interlocutores la cual se da cuando se exige que lo realizado por el antropólogo en términos de los resultados de investigación, sea un libro, un texto o una producción audiovisual, sea conocido y socializado oportunamente por el mismo investigador ante la comunidad donde realizó la investigación. 
Desde esta interpretación el imaginario del poder de los libros y su reconocimiento público, financiero o no, constituyen una de las principales aspiraciones de beneficiarse que tienen los interlocutores. Sin embargo, existe una segunda variante interpretativa que no se funda en el sistema de valores que usualmente los académicos tenemos para transmitir ideas y conocimientos a través de libros o productos, y tiene que ver con otras formas de prestación de favores y reciprocidades propias de las mismas dinámicas locales y el espíritu de servir por parte del mismo antropólogo/a ${ }^{12} 11$. Esta variante se funda en la representación proyectada de que al provenir del "mundo de afuera" el antropólogo dispone de herramientas, recursos, "contactos" que pueden servir a la comunidad en la resolución de asuntos propios de ellos como aspectos organizativos, salud, educación, el papeleo, trámites en las ciudades anexas o ciudades capitales o ciertos favores que implican un conocimiento básico del medio que se supone el/la antropólogo/a conoce. Desde esta perspectiva, los líderes pueden consolidar su poder a través del logro de bienes y servicios que pueden demandarle a los investigadores, quienes a su vez pueden ser vistos como contactos importantes en redes de relaciones más amplias (BOISSEVAIN 1979; 1968; SCHMIDT 1977; TOCANCIPÁ-FALLA 2013; WOLF 2001).

En tercer lugar, la reciprocidad no es un asunto o problema exclusivamente de los antropólogos, sino también del sentido común de la gente en general. La historia del encuentro colonial está llena de ejemplos con los misioneros y colonizadores. El caso de Puyo, capital de Pastaza en la amazonía ecuatoriana no es la excepción, tal como lo referencia el historiador Zamora (2009b) con respecto a la situación de llegada de Lucindo, uno de los primeros colonizadores a la región a comienzos del siglo XX:

\begin{abstract}
"Poco a poco comenzaron a sentir los efectos de la dura realidad, claro que era una tierra rica, pero a la vez guardiana de sus riquezas (...) Lucindo haciendo uso de las pocas palabras de quichua aprendidas en la estancia anterior en Canelos, les hizo comprender, de la necesidad que tenían de que les brinden posada, para que descansen sus desfallecidos cuerpos, mucho más cuando entre ellos había niños de corta edad. La tarea no fue fácil, pero, entregándoles objetos llamativos y novedosos para los indígenas, lograron sensibilizarlos, hasta que accedieron a darles posada, aunque el hambre hacía presa de la desesperación pudieron descansar" (ZAMORA, 2009b, p.sf.).
\end{abstract}

Finalmente, existe una lección importante en el caso examinado sobre las alusiones a que los antropólogos sean más recíprocos con sus pares "otros" en campo, y tiene que ver con las implicaciones de este análisis en una perspectiva teórica-metodológica en la cual el/la antropólogo/a se ve inmerso en una experiencia investigativa en campo. Una de estas

\footnotetext{
${ }^{12}$ Este es el caso de los Whitten quienes con el acompañamiento de Marcelo F. Naranjo, un kichwa de la provincia de Pastaza, crearon The Sacha Runa Research Foundation con el fin de establecer un marco de cooperación con los kichwas de Canelos y los achuarde la provincia de Pastaza sobre la base de contribuir en la atención médica de los comuneros a cambio de ofrecer cooperación en procesos de investigación, y que en muchos casos incluyó la divulgación y promoción de estos grupos a través de la producción alfarera, entre otras actividades, existente en la región (WHITTEN; WHITTEN, 1977). De esta manera se trataba de una especie de trueque entre investigación y servicios en salud y promoción de la cultura material.
} 
implicaciones es la creciente necesidad porque los antropólogos involucren más a sus pares "otros" en la identificación y formulación de problemas de investigación. Si se presume que nuestra disciplina es una carrera humanística no se logra comprender cómo todavía se excluyen a los actores sociales de esa posibilidad. No se trata de convertir esta posibilidad en una generalidad pero sí un reconocimiento de que los problemas antropológicos e interrogantes identificados sean más afines a las realidades estudiadas como tal. Involucrar a los actores sociales en el proceso de investigación desde sus inicios puede omitir pasos y procesos obligados como "socializar" o "divulgar los resultados de investigación", categorías comunes en los formatos estatales que formulan las agencias estatales que apoyan la investigación social. En últimas, se trata de generar espacios de interacción donde los estudiosos de lo social y los mismos actores sociales puedan generar un conocimiento en prospectiva con una mayor pertinencia y correspondencia con las condiciones locales que viven los actores. Esta idea nos lleva a pensar en posibilidades de un conocimiento situado en prospectiva.

\section{CONOCIMIENTO ANTROPOLÓGICO SITUADO EN PROSPECTIVA}

Por conocimiento situado en prospectiva entendemos aquel conocimiento que ha sido producido en relación dialógica y dialéctica entre investigadores y actores sociales que comparten el entorno social que es indagado o investigado; y que se convierte en el fundamento de pesquisas posteriores que escalonadamente van ofreciendo interpretaciones o explicaciones de hechos no examinados con anterioridad, o que si lo han sido, presentan nuevas interpretaciones o explicaciones antes no previstas. Lograr esto, sin embargo, requiere un esfuerzo y dedicación en términos de lograr un espacio físico y simbólico que permita el desarrollo de estas ideas.

La experiencia alcanzada en la amazonía ecuatoriana posibilitó un primer acercamiento que, como indicamos al comienzo de este trabajo, involucró a líderes de las 7 nacionalidades existentes en la amazonía ecuatoriana, con quienes se reconoció parte de la problemática ya examinada en la primera parte de este trabajo sobre la importancia de ser recíprocos en la producción del conocimiento antropológico.

Una primera iniciativa, muy preliminar y formulada desde el ámbito académico, fue instalar una base de datos que se denominó Binahme: Base Bibliográfica de Investigaciones históricas y antropológicas de la amazonía ecuatoriana, la cual cuenta en la actualidad con más de cien entradas de investigaciones antropológicas realizadas por antropólogos de las nacionalidades (Ver www.uea.educ.ec). La meta es contar por lo menos con siete subgrupos organizados de investigaciones por cada nacionalidad, con el fin de encontrar líneas de trabajo 
conjuntas con cada una de ellas sobre temas y problemas correspondientes a su situación actual. Se trata de un proyecto de largo aliento que al final busca delimitar y establecer agendas de investigación no solo antropológicas sino también interdisciplinarias y en otros campos de la investigación social.

A la par de esta plataforma de trabajo se viene avanzando igualmente en premisas o principios de investigación social que se basan en lo discutido aquí sobre la reciprocidad en la investigación y desarrollos conjuntos de investigaciones colaborativas y de intercambio de experiencias y conocimientos. Como tal, estas dos iniciativas no son en definitiva una salida conclusiva a una problemática creciente y compleja, que también involucra otros campos de conocimiento, pero que al menos significa un pequeño avance hacia la ruptura con un modelo de investigación extractivo y que en su lugar se propenda por una participación más activa y dialógica con aquellos que se suponen hacen parte de nuestra experiencia de vida y de conocimiento.

\section{ALGUNAS CONSIDERACIONES FINALES}

Históricamente la Amazonía, en particular la ecuatoriana, ha sido considerada como una fuente importante de recursos de distinto orden. Hemos visto que alrededor de esta idea se tejen diversas formas de representación social y que en este caso hemos abordado por lo menos dos de ellas que aluden a esta vasta región como "Tierra de promisión" y "Tierra de promesas incumplidas"; representaciones coexistentes en la actualidad y que tensionan las relaciones entre el Estado y los movimientos sociales indígenas. Frente a la riqueza biodiversa de la amazonía, tanto en términos de recursos naturales y culturales, se ha destacado la importancia de documentar e investigar estas realidades sobre la base de que allí se pueden encontrar elementos transformadores para la sociedad ecuatoriana, pero también se pueden hallar acervos representados en conocimientos y prácticas culturales e interculturales que todavía se mantienen desde hace centurias.

Es en este ámbito, y a partir del ingreso en el contexto amazónico ecuatoriano, que hemos sugerido la trayectoria de un conocimiento en retrospectiva y un conocimiento en prospectiva, que involucra 'otras' reciprocidades en las cuales el antropólogo se ve inmerso en su proceso de aceptación en campo. Desde esta perspectiva se ha sugerido que estas trayectorias tienen implicaciones de tipo teórico-metodológico que pueden anticipar otras maneras, seguramente ya avanzadas en otros contextos, de realizar investigación; una más colaborativa y participativa a otra convencional y "extractiva" que se ha venido practicando en otros ámbitos amazónicos. En esta línea, pueden plantearse varias conclusiones y algunos interrogantes para posteriores trabajos. 
En cuanto a las conclusiones, se ha notado que, en primer lugar, es importante documentar la manera como en el pasado los antropólogos han ingresado a la escena cultural y han desarrollado relaciones diversas a través de colaboraciones, compromisos y actividades que a la mirada de los interlocutores han sido significativas o por lo menos perdurables en la memoria. El caso de los Whitten que trabajaron en la amazonía ecuatoriana, en particular en Pastaza, donde crearon una Fundación para favorecer la comercialización de mukawas y con los recursos ayudaron a las comunidades kichwas y achuar para afrontar temas de salud y divulgación de la cultura material, es un buen ejemplo de ello.

En segundo lugar, y a partir de lo anterior, se ha notado que la presencia de los antropólogos, entre otros investigadores, deja una "huella" de su participación en las comunidades que en algunos casos aparece imborrable, bien sea por el servicio prestado, en la comunidad, por tener un interés en un tema que la comunidad no había apreciado o, bien sea porque se trataron de reciprocidades no cumplidas, o por las dos anteriores. Como notamos en nuestro caso, esta huella sale a relucir bien, sea como una estrategia de negociación para el recién llegado en los términos de los compromisos que se van a adquirir o bien sea porque, en el fondo, este es visto como alguien que puede tener contactos importantes con el mundo exterior. En cualquier caso, se ha notado que es importante que el/la antropólogo/a que se inicia en el trabajo de campo establezca los términos claros de cooperación y el verdadero alcance y cumplimiento de los compromisos que se van a adquirir.

En tercer lugar, se destaca que las implicaciones de conocer la trayectoria de un conocimiento en retrospectiva son de orden teórico-metodológico. Son de orden teórico porque un diálogo sostenido con los interlocutores en campo sobre las preocupaciones académicas iniciales puede afinar el problema, el uso de los conceptos y la perspectiva teórica que se estima proyectar en la investigación. Pero también puede implicar que en este diálogo se visualicen otros campos problemáticos no antes cartografiados desde la academia y que muchas veces resultan en problemas de investigación. De otro lado puede tener implicaciones metodológicas por cuanto supone establecer los términos de relación para establecerse en la investigación en campo y lograr un trabajo colectivo que se oriente a la búsqueda de las respuestas propuestas en la misma investigación. En este escenario se invocan los compromisos, productos y resultados esperados donde seguramente pueden haber exigencias por parte de los líderes que tendrán que ser evaluadas. En cualquier caso, el punto central de la argumentación es que el antropólogo que se inicia recientemente en el trabajo de campo debe ganar una mayor consciencia que realizar investigación en campo significa estimar el tipo de reciprocidades en las cuales se ve expuesto/a de manera directa o indirecta. Muchas veces el poder de la dinámica local donde se encuentran varias necesidades puede ser un gran remolino y el/la antropólogo/a puede terminar absorbido (a). He ahí el gran reto que tiene para establecer hasta dónde llega y hasta dónde no puede llegar. 
En cuanto a los interrogantes, merecen enunciarse algunos que pueden servir para futuras investigaciones: ¿En un contexto altamente politizado, hasta qué punto la participación del antropólogo o de la antropóloga debe ser sostenida y visible?¿en qué fases del trabajo de campo, es necesario precisar una participación de este orden? ¿Qué implicaciones tiene la colaboración recíproca en un posible acrecentamiento del poder de algunos líderes o actores sociales en detrimento de otros?:Teniendo en cuenta una perspectiva de género y de posibilidades de realización, en qué términos se pueden plantear unas relaciones de intercambio recíproco?

Finalmente, estas conclusiones e interrogantes, convergen en un aspecto básico que la antropología en América Latina ha ido perfilando en los últimos años sobre su papel en las sociedades donde se desenvuelve y el nivel de compromiso que debe asumir. Significa ganar una mayor sensibilidad y afinidad en las relaciones e interacciones que mantenemos con nuestros interlocutores. Es claro que al respecto no existen fórmulas, aunque sí lineamientos que pueden servir a futuras generaciones de antropólogos y antropólogas para continuar perseverando en construir un espacio y puente más interactivo, y menos quizás, fundamentalista en la reificación de los esencialismos. 


\section{REFERENCIAS}

BENEDICT, Anderson. Imagined Communities: Reflections on the Origin and Spread of Nationalism. London: Verso. 1996

BECKER, Marc. ¡Pachakutiki: Movimientos indígenas, proyectos políticos y disputas electorales en el Ecuador. Quito: Flacso Abya Yala, 2015.

BOISSEVAIN, Jeremy. The place of non-groups in the social sciences. Man, New Series, 1968. p.3(4):542556. Network analysis: a reappraisal. Current Anthropology, 1979. p.20(2):392-94.

BOURDIEU, Pierre. Outline of a Theory of Practice. R. Nice, transl. Cambridge: Cambridge University Press, 1977.

CLIFFORD, James; MARCUS, George E. Writing culture: the poetics and politics of ethnography: Berkeley: University of California Press, 1986.

CONSTITUYENTE, Asamblea. Constitución del Ecuador. Montecristi, Ecuador: Asamblea Constituyente, 2008.

DUVEEN, Gerard. LLOYD, Barbara. Social Representations and the Development of Knowledge. Cambridge: Cambridge University Press, 1990.

HURTADO, Enrique. Para el Ministro del Interior. G.d.0.-R.d. Ecuador, ed.p. 1-4. Archidona, 24 de agosto, 1899a.

. Señor Ministro de lo Interior y Policía encargado de la sección de obras públicas. G.d.l.p.o.-R.d. Ecuador, ed. Pp. 1-2. Archidona, 28 de enero, 1899 b.

INES, Instituto Nacional de Estadisticas y Censo - 2015 Censo 2010. Quito http://www.ecuadorencifras.gob.ec/: Unidad de Procesamiento - Dirección de Estudios Analíticos Estadísticos, 1899.

JENKINS, Richard. Social Identity. London \& New York: Routledge, 1996.

LÉVI-STRAUSS, Claude. Introducción. In: MAUSS, M. (org). Sociologia y Antropología. Madrid: Tecno, 1979. p. 13-42.

MAUSS, Marcel. The Gift. The form and reason for exchange in archaic societies. London: Routledge, 1990.

NEVES, Eduardo. PETERSEN, J. BARTONE R. SILVA, C. Historical and Socio - cultural Origins of Amazonian Dark Earths. In: LEHMANN, J.(Org.) Amazonian Dark Earths: Origin, Properties, Management. Netherlands: Kluwer Academic Publishers, 2003. p. 29-50.

POOLE, Ross. Nation and Identity. London: Routledge, 1999.

ORAPPORT, Nigel. OVERING, Joanna. Auto-Anthropology. In: RAPPORT, N. OVERING, J. Social and Cultural Anthropology. The Key concepts. Oxon: Routledge, 2007. p. 19-30. 
SAWYER, Suzana. Crude Chronicles: Indigenous politics, multinational oil, and neoliberalism in Ecuador. Durham \& London: Duke University Press, 2004.

SCHMIDT, W. Steffen. The transformation of clientelism in rural Colombia. In: W.S. Schmidt (Org). Friends, Followers and Factions: A reader in Political Clientelism. Berkeley,Los Angeles e Londres: University of California Press, 1977, p. 305-323.

SENPLADES, Secretaría Nacional de Planificación y Desarrollo - Plan Nacional de Desarrollo / Plan Nacional para el Buen Vivir 2013-2017. Senplades, ed. Quito, 2013.

TAUSSIG, Michael. Chamanismo, colonialismo y el hombre salvaje. Un estudio sobre el terror y la curación. Popayán: Universidad del Cauca, 2002.

TAYLOR, Anne Christine. The Western margins of Amazonia from the early sixteenth to the early nineteenth century. In: SALOMON, F. SCHWARTZ, S.B. The Cambridge history of the native peoples of the Americas. Cambridge: Cambridge University Press, v. 3, 1999, p. 188-256.

THORNTON, Robert. The rhetoric of ethnographic holism. Cultural Anthropology, v. 3 p. 285-303, 1988.

TOCANCIPÁ-FALLA, Jairo. Campesinos, Brokers and Politicians: Revisiting the Power of Exchanging Favours in Local Colombian Politics. International Journal of Humanities and Social Science, v. 3 p.6174, 2013.

WEISS, Joseph. BUSTAMANTE, Teodoro. Ajedrez ambiental: Manejo de recursos naturales, comunidades, conflictos y cooperación. Quito: FLACSO y Ministerio de Cultura, 2008.

WHITTEN, Dorothea. WHITTEN, N. Collaborative Research. Report on the Sacha Runa Research Foundation. Medical Anthropological Quarterly, p. 4-5, 1977.

WHITTEN, Norman. NARANJO, M. SIMBANA, M. WHITTEN, D. Sacha Runa: ethnicity and adaptation of Ecuadorian jungle Quichua: University of Illinois, 1977.

WOLF, Eric. Kinship, friendship, and patron-client relations in complex societies. In: WOLF. E. Pathways of Power: Building an Anthropology of the Modern World. Berkeley; Los Angeles; London: University of California Press, 2001, p. 166-183.

WOOLGAR, Steve. Reflexivity. In: KUPER, A. KUPER, J. (0rg.) The Social Science Encyclopaedia. London and New York: Routledge, 1996. p. 725- 726.

ZAMORA, Oscar. Pastaza: Una provincia que apasiona. Puyo: Consejo provincial de Pastaza, 2004.

El pasado en el presente de Puyo: 1930-1960. Tomo II. Puyo: Editorial CCE-NP, 2009a. História viva de Pastaza 50 años. Puyo: Editorial CCE - NP, $2009 \mathrm{~b}$. 